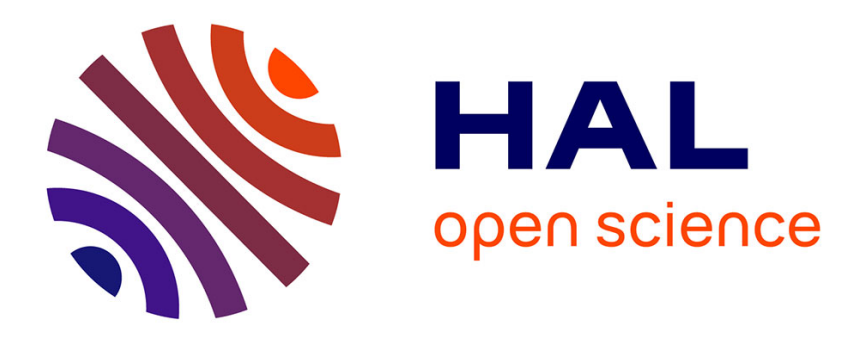

\title{
Comportement vibratoire de réseau de plaques assemblées
}

E. Rebillard, Jean-Louis Guyader

\section{To cite this version:}

E. Rebillard, Jean-Louis Guyader. Comportement vibratoire de réseau de plaques assemblées. Journal de Physique IV Proceedings, 1994, 04 (C5), pp.C5-121-C5-124. 10.1051/jp4:1994520 . jpa-00252883

\section{HAL Id: jpa-00252883 https://hal.science/jpa-00252883}

Submitted on 1 Jan 1994

HAL is a multi-disciplinary open access archive for the deposit and dissemination of scientific research documents, whether they are published or not. The documents may come from teaching and research institutions in France or abroad, or from public or private research centers.
L'archive ouverte pluridisciplinaire HAL, est destinée au dépôt et à la diffusion de documents scientifiques de niveau recherche, publiés ou non, émanant des établissements d'enseignement et de recherche français ou étrangers, des laboratoires publics ou privés. 


\title{
Comportement vibratoire de réseau de plaques assemblées
}

\author{
E. REBILLARD et J.-L. GUYADER
}

L.V.A., Bâtiment 303, INSA de Lyon, 20 Avenue Albert Einstein, 69621 Villeurbanne cedex, France

\begin{abstract}
résumé :The aim of this work is to explain the vibrational behaviour of several plate structures whose plates are joined at different angles, having same widths but different lengths. An analytical formulation using a semi modal decomposition along the junction direction and a wave formulation along the perpendicular one is developed. This approach is very powerful because its numerical implementation is not too heavy (contrary to FEM) and because it's very versatile. Basic vibrational control parameters such as the quadratic velocity and the transfert mobility are shown. The simplest structure considered in this study is made of twin plates connected at any angle. It is shown that in some particular configuration, the vibrational behaviour of the structure can be highly sensitive to slight modifications of a geometrical parameter, particularly the angle between the plates. A statistical way is used to illustrate this phenomenon currently encountered in industrial applications. This formulation can also deal with periodic structures such as corrugated plates.
\end{abstract}

\section{INTRODUCTION - MODELE ETUDIE}

De nombreuses études se sont interessées au problème de la vibration de plaques couplées en flexion. La plupart des modèles sont restreints quant au nombre de plaques $(2,3$ ou 4$)$ et quant à la disposition, forme en $T$, en $L$, ou en croix $[1,2,3]$.

C'est pourquoi le modèle analytique présenté ici définit le comportement vibratoire d'un assemblage de plaques d'identique largeur mais de longueurs différentes, assemblées à angle quelconque, où les effets de flexion et de membrane sont considérés.

Les conditions aux limites sur les bords qui ne font pas office de liaison sont modélisées comme des appuis simples. La structure est excitée par une force ponctuelle harmonique de direction et de position quelconque mais choisie.-cf figure 1 -

\section{VIBRATION DES PLAQUES COUPLEES}

Trois points-clefs constituent la base de la résolution analytique de ce problème.

*Pour obtenir les mouvements de flexion et de membrane d'une plaque, on peut la considérer comme une coque de rayon infini, et utiliser l'opérateur de Donnell. Au sein de chaque plaque, les effets de flexion et de membrane sont découplés.

$$
\frac{E h}{1-v^{2}} L_{\infty}\left[\begin{array}{c}
u \\
v \\
w
\end{array}\right]-\rho h \frac{\partial^{2}}{\partial t^{2}}\left[\begin{array}{c}
u \\
v \\
w
\end{array}\right]=\varepsilon \delta(x-x 0) \delta(y-y 0)\left[\begin{array}{l}
F_{x} \\
F_{y} \\
F_{z}
\end{array}\right]
$$

où $E$ est le module de Young, $v$ le coefficient de Poisson, $\rho$ la masse volumique, h l'épaisseur de la plaque. $\varepsilon$ vaut 1 si la plaque est excitée par la force de composantes $\mathrm{Fx}$, Fy et $\mathrm{Fz}$ appliquée en $\mathrm{x} 0$ et y0 et vaut zéro 
sinon. $L_{\infty}$ est l'opérateur de Donnell pour un rayon de coque infini; pour un rayon fini, il est exprimé en $[4]$. forme:

* Une décomposition modale dans la direction y permet d'exprimer les trois déplacements sous cette

$$
u(x, y)=\sum_{n=1}^{\infty} U_{n}(x) \sin \frac{n \pi y}{a}, v(x, y)=\sum_{n=0}^{\infty} V_{n}(x) \cos \frac{n \pi y}{a}, \quad w(x, y)=\sum_{n=1}^{\infty} W_{n}(x) \sin \frac{n \pi y}{a}
$$

où $n$ est l'indice modal de la décomposition.

Chaque $W_{n}(x)$ (respectivement chaque couple $U_{n}(x), V_{n}(x)$ ) est solution d'une équation différentielle (respectivement d'un système de deux équations différentielles) et la solution adopte un formalisme d'onde de la forme:

$W_{n}(x)=A_{n} e^{-k_{n 1} x}+B_{n} e^{-k_{n 2} x}+C_{n} e^{-k_{n 1}(1-x)}+D_{n} e^{-k_{n 2}(l-x)}$

où $k_{n 1}$ et $k_{n} 2$ sont les nombres d'onde de flexion et $A_{n}, B_{n}, C_{n}, D_{n}$ sont les amplitudes inconnues.

* Le couplage entre deux plaques est exprimé par la continuité à la jonction de huit grandeurs: les trois déplacements, les trois efforts, le moment fléchissant et la rotation le long de l'axe de jonction. L'écriture des huit conditions à chaque liaison et des conditions aux limites des plaques extrèmes donne naissance à un système matriciel couplant les effets de flexion et de membrane de deux plaques voisines, dont les inconnues sont $A_{n}, B_{n}, C_{n}, D_{n}$ (liés aux mouvements de flexion) et quatre autres coefficients équivallents liés aux mouvements dans le plan. Cette résolution est faite pour chaque mode $n$.

La reconstruction des déplacements totaux d'après l'équation (2) permet de définir les paramètres de contrôle vibratoire comme la vitesse quadratique de plaque ou de toute la structure, ou comme la mobilité de transfert.

\section{APPROCHE STATISTIQUE DES EFFETS DE DEFAUTS}

On peut considérer une population de structures industriellement identiques, formées de deux plaques inclinées, dont un paramètre (l'angle entre les deux plaques) suit une distribution gaussienne (de moyenne $\bar{\theta}$ et d'écart type $\sigma$ ) modélisant un défaut. Les paramètres statistiques liés à la mobilité de transfert des structures de la population sont obtenus soit par voie numérique: méthode de type Monte-Carlo, soit par voie analytique.

La démarche analytique considère la mobilité de transfert entre deux points $A$ et $B$ de la structure comme une fonction de l'angle entre les deux plaques, on la note $\mathrm{Y}(\mathrm{A}, \mathrm{B}, \theta)$; elle est exprimée par une décomposition en série de Fourier.

$\bar{Y}(A, B, \theta)=\sum_{m=0}^{M} \alpha_{m} \cos m x+\beta_{m} \sin m x$

On peut alors exprimer de façon analytique les paramètres statistiques de $\mathrm{Y}(\mathrm{A}, \mathrm{B}, \theta)$; on montre que la moyenne et la variance (qui vaut le carré de l'écart-type) ont respectivement pour forme:

$$
\begin{aligned}
\bar{Y}(A, B, \theta)= & \sum_{m=1}^{M} \alpha_{m} e^{-\left(\frac{m^{2} \sigma^{2}}{2}\right)} \\
S^{2}(Y(A, B, \theta))= & \frac{1}{2} \sum_{m}^{M} \sum_{q}^{Q} \alpha_{m} \alpha_{q}\left(e^{-\frac{(m+q)^{2} \sigma^{2}}{2}}+e^{-\frac{(m-q)^{2} \sigma^{2}}{2}}\right)+\frac{1}{2} \sum_{m}^{M} \sum_{q}^{Q} \beta_{m} \beta_{q}\left(e^{-\frac{(m-q)^{2} \sigma^{2}}{2}}+e^{-\frac{(m+q)^{2} \sigma^{2}}{2}}\right) \\
& -\sum_{m}^{M} \sum_{q}^{Q} \alpha_{m} \beta_{q} e^{-\frac{\left(m^{2}+q^{2}\right) \sigma^{2}}{2}}
\end{aligned}
$$




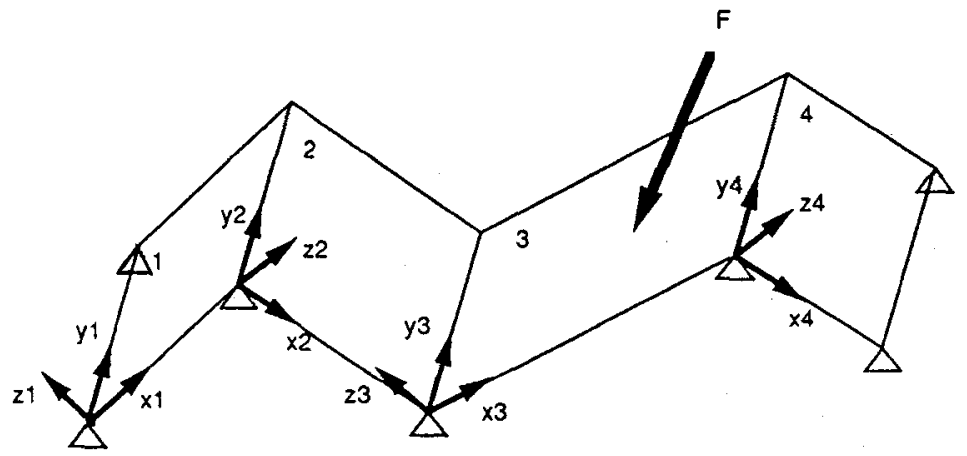

fig. 1 Exemple de structure etudiee
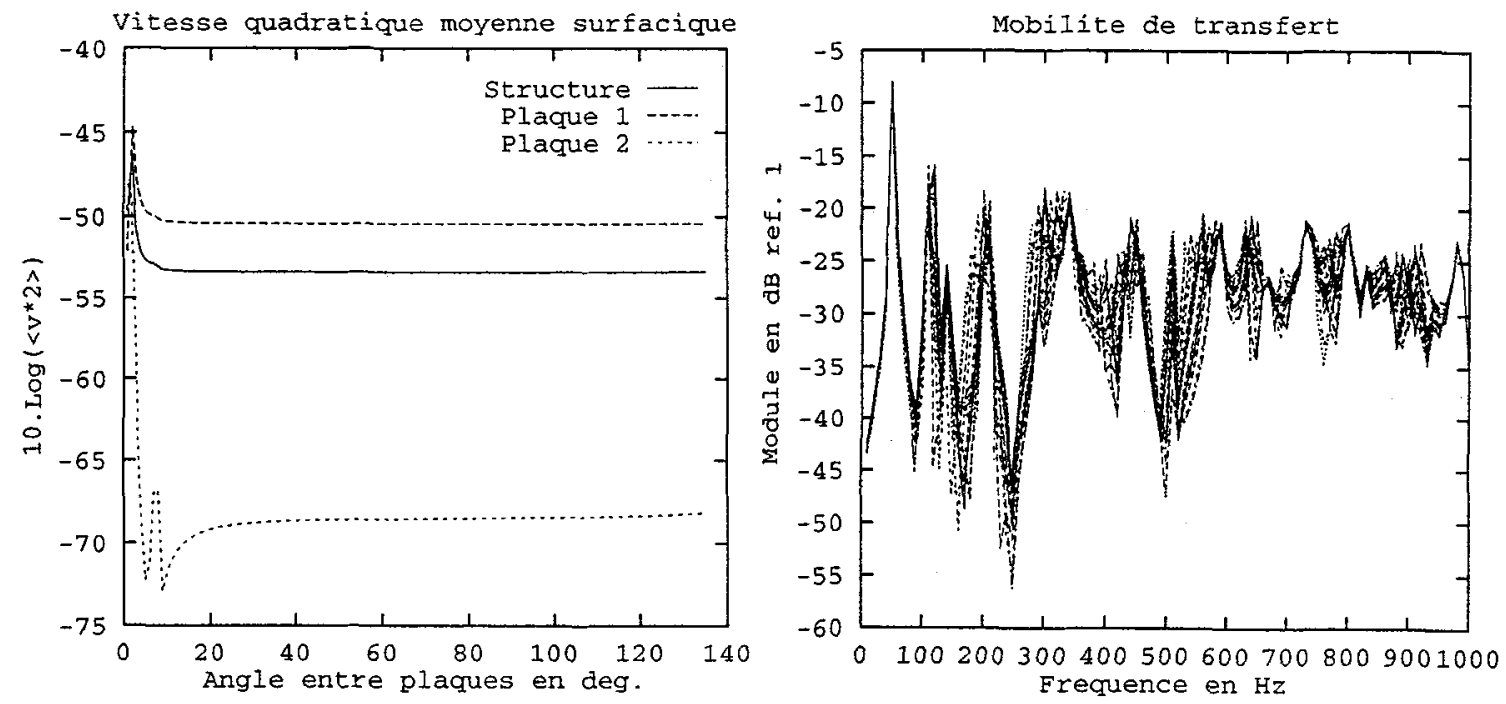

fig. 2

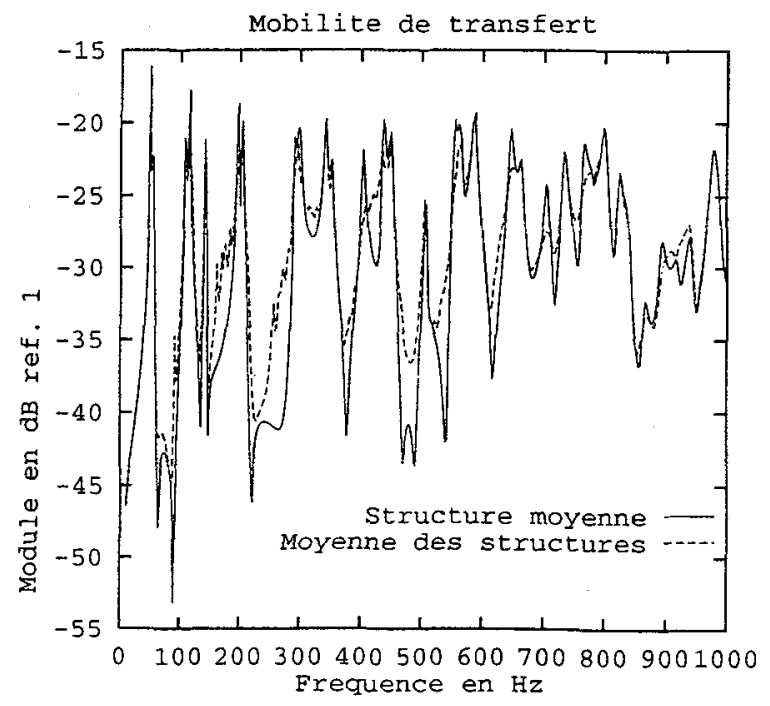

fig. 3

fig. 4 


\section{EXEMPLES DE RESULTATS}

Le premier exemple considère 2 plaques en acier (de dimensions: $0.5 \times 0.4 \times 0.002 \mathrm{~m}$ ) identiques dont l'angle de couplage varie. On met en évidence, dans certaines configurations, une grande sensibilité du comportement vibratoire pour une faible variation du paramètre angulaire. Ce fait est illustré aux figures 2 et 3.

A la figure 2 est présentée la variation de la vitesse quadratique de chacune des plaques et de toute la structure, à fréquence fixée, mais pour l'angle entre les plaques variant. Pour certains angles faibles, la variation de la vitesse est très grande pour une très faible variation de l'angle.

A la figure 3 on considère une population de trente structures formées de deux plaques identiques (de mêmes dimensions que précédemment) couplées à un angle $\theta=\theta_{0}+\varepsilon$ (où $\theta_{0}$ vaut 4 degrés et e est une variable aléatoire gaussienne de moyenne nulle et d'écart type 1); les spectres de mobilité de transfert montrent que pour une faible variation angulaire, une forte variation du comportement peut apparaître. Une telle approche est à mettre en rapport avec des travaux proposés par F.Fahy [5].

Le traitement statistique du comportement vibratoire d'une telle population de structures permet de mettre en évidence que le comportment moyen de toutes les structures est différent du comportement de la structure moyenne. Ceci est illustré par les spectres de mobilité de transfert à la figure 4 .

\section{CONCLUSION}

Cette méthode analytique puissante quant au potentiel de modélisation et quant à la rapidité calculatoire permet de mettre en évidence une forte modification du comportement vibratoire de structures formées de deux plaques couplées pour une faible variation paramètrique, sous certaines conditions. Elle permet aussi, entre autre, d'étudier des structures de type réseau de plaques éventuellement périodique. Une continuité de ce travail est d'incorporer la notion de défaut (ou faible variation paramètrique) aux structures périodiques.

\section{Références:}

[1] J.L. Guyader, C.Boisson and C.Lesueur, 1982 JSV 81(1) 81-92

[2] C.Boisson, J.L.Guyader, P.Millot and C.Lesueur, 1982 JSV 81(1) 93-105

[3] B.M.Gibbs and Y.Shen, 1987 JSV 112(3) 469-485

[4] Leissa (A.W), Vibration of shells, NASA's publications, Washington DC, 1973

[5] F.Fahy, Inter Noise 1993 Leuven Belgium. August 24-26 Vol I 13-26

Statistical Energy Analysis: A wolf in sheep's clothing?

\section{Remerciements:}

Ce travail est réalisé dans le cadre d'une thèse de doctorat financée par une bourse DRET-CNRS. 